\title{
High resolution simulations of unstable modes in a collisionless disc
}

\author{
A. V. Khoperskov ${ }^{1,2}$, A. Just ${ }^{3}$, V. I. Korchagin ${ }^{4,5}$, and M. A. Jalali ${ }^{6}$ \\ 1 Department of Theoretical Physics, Volgograd State University, Volgograd 400068, Russia \\ 2 Isaac Newton Institute of Chile, Moscow Branch, Russia \\ 3 Astronomisches Rechen-Institut at ZAH, University of Heidelberg, Mönchhofstraße 12-14, 69120 Heidelberg, Germany \\ e-mail: just@ari.uni-heidelberg.de \\ ${ }^{4}$ Institute of Physics, Stachki 194, Rostov-on-Don 344090, Russia \\ 5 Isaac Newton Institute of Chile, Rostov-on-Don Branch \\ ${ }^{6}$ Department of Mechanical Engineering, Sharif University of Technology, Azadi Ave., Tehran, Iran
}

Received 5 October 2006 / Accepted 3 July 2007

\section{ABSTRACT}

\begin{abstract}
We present $N$-body simulations of unstable spiral modes in a dynamically cool collisionless disc. We show that spiral modes grow in a thin collisionless disk in accordance with the analytical perturbation theory. We use the particle-mesh code SUPERBOX with nested grids to follow the evolution of unstable spirals that emerge from an unstable equilibrium state. We use a large number of particles (up to $N=40 \times 10^{6}$ ) and high-resolution spatial grids in our simulations ( $128^{3}$ cells). These allow us to trace the dynamics of the unstable spiral modes until their wave amplitudes are saturated due to nonlinear effects. In general, the results of our simulations are in agreement with the analytical predictions. The growth rate and the pattern speed of the most unstable bar-mode measured in $N$-body simulations agree with the linear analysis. However the parameters of secondary unstable modes are in lesser agreement because of the still limited resolution of our simulations.
\end{abstract}

Key words. stellar dynamics - galaxies: kinematics and dynamics - instabilities - galaxies: structure

\section{Introduction}

Gravitational instability of galactic discs is a widely accepted physical mechanism for the generation of barred and spiral structures in disc galaxies. Since the pioneering publications by Lin \& Shu (1964) and Toomre (1981), considerable progress has been made in the theoretical study of gravitational instabilities of disc-like systems. A steady stream of papers performing a linear stability analysis of self-gravitating gaseous discs (e.g., Bertin et al. 1989a,b; Adams et al. 1989; Savonije \& Heemskerk 1990) have demonstrated that a massive gaseous disc will almost inevitably be prone to spiral instabilities. A number of numerical techniques have been applied to study the nonlinear evolution of unstable gaseous discs (e.g., Tomley et al. 1994; Miyama et al. 1994; Woodward et al. 1994; Nelson et al. 1998; Laughlin et al. 1997,1998). Recently, the linear approach has been generalized for the stability analysis of non-axisymmetric gaseous disks (Asghari \& Jalali 2006). These contributions address the dynamical modeling of gaseous discs with a variety of equilibrium properties and compare the results of numerical simulations with those of analytical predictions.

The dynamical study of the collisionless gravitating discs is hampered by the difficulty of normal mode calculations in such systems. A global modal analysis of collisionless discs has been accomplished for a few cases. Kalnajs (1972) calculated the global modes of a uniformly rotating, self-gravitating stellar disc. Zang (1976) carried out a modal analysis of Mestel's stellar disc using an inner cutout function to handle the central singularity. His method was then used by Evans \& Read (1998a,b) for a general class of scale-free discs with rising and falling rotation curves. The modal properties of a collisonless exponential disc (with a core) were also studied by Vauterin \& Dejonghe (1996). Most recently, Jalali \& Hunter (2005, hereafter JH) developed a rather general approach that allowed them to calculate the modal properties of stellar discs. They showed that the radial orbits of soft-centered models lead to a boundary integral that plays a crucial role in the formation and growth of a bar mode, which is localized within the central regions of a collisionless disc. They also investigated the effect of dark halos/bulges on the properties of growing modes.

The major drawback of perturbation theories is that they cannot make any prediction of modal evolution in the nonlinear regime. A linear analysis cannot predict the saturation level of the wave amplitude after an exponential growth phase, nor can it predict the duration of the linear and nonlinear phases. One can tackle these problems by using $N$-body simulations.

An $N$-body approach has a number of difficulties, such as numerical relaxation due to a high level of noise and finite spatial resolution. These factors hamper the behavior of collisionless models. Moreover, growing modes are sensitive to the equilibrium profiles of the central regions. Thus, numerical simulations with a large number of particles, and high spatial resolution, are needed to correctly model the behavior of unstable modes in collisionless discs.

There have been a few attempts to numerically model the dynamics of global modes in collisionless discs. As already mentioned, modeling of the spiral structure in collisionless galaxies and comparison with analytical predictions has been restrained by the relatively small number of particles used in $N$-body codes. Randomly generated initial coordinates of particles inevitably lead to density fluctuations and perturbing forces that affect the 
disc dynamics (Sellwood 1983). The disc response to these virtual forces could contribute substantial errors of about 50 percent to the computed growth rates and the pattern speeds (Sellwood 1983). In previous $N$-body simulations aimed at studying the dynamics of global modes in collisionless discs (Athanassoula \& Sellwood 1986; Sellwood \& Athanassoula 1986), special precautions have been taken to avoid a strong influence of noise perturbations. These authors used "quiet" initial conditions, with the groups of stars spaced at equal intervals around the rings at fixed radii, and all particles in a ring were given the same initial radial and tangential velocity components.

The development of numerical techniques with tens of millions of particles would avoid artificial precautions in tackling the numerical noise problem. In this paper, we use SUPERBOX (Fellhauer et al. 2000) to model the evolution of a collisionless disc. SUPERBOX is a highly efficient particle-mesh-code with nested and co-moving grids, based on a leap-frog scheme designed for the simulation of interacting galaxies or other stellar systems. Aiming at modeling the dynamics of spiral patterns in real galaxies, we apply the code to analyse one of $\mathrm{JH}$ models that has an exponential disc density distribution with a core, embedded in a cored logarithmic potential. We use this model for two reasons. Firstly, closed-form expressions are available for the phase space distribution functions. Secondly, a full spectrum of unstable modes has been computed for this model.

We find good agreement between the linear global modal analysis and the nonlinear simulations. The growth rate and the pattern speed of the most unstable $m=2$ bar-like global mode found in $N$-body simulations, and the spatial distribution of this mode, agree well with the analytical results. We have also been able to recover the theoretical growth rates and the pattern speeds of the unstable $m=3$ and $m=4$ global modes. Apart from demonstrating the existence of global modes in the cored exponential discs, our simulations provide a welcome check of the SUPERBOX code, and demonstrate its applicability to model the dynamics of real galaxies.

In Sect. 2 we briefly summarize the properties of the global modes of $\mathrm{JH}$ models, and use their method to find the unstable $m=2, m=3$ and $m=4$ modes of a cored exponential disc. In Sect. 3 we describe the $N$-body code and its results for the model of $\mathrm{JH}$. A comparison of the results of $N$-body simulations with those of analytical predictions is given in the same section. Section 4 contains a summary of our results.

\section{The analytical model}

We study the stability properties of a razor-thin disc that has an exponential density distribution with a central core of radius $R_{\mathrm{C}}$ :

$\Sigma_{\mathrm{D}}(R)=\Sigma_{0} \exp \left(-\lambda \sqrt{1+R^{2} / R_{\mathrm{C}}^{2}}\right), \quad \lambda=\frac{R_{\mathrm{C}}}{R_{\mathrm{D}}}$.

We assume that the disc is in equilibrium with the total gravitational potential of the galaxy represented by the logarithmic law

$\Phi_{0}(R)=v_{0}^{2} \ln \sqrt{1+R^{2} / R_{\mathrm{C}}^{2}}$.

Here $\Phi_{0}(R)$ is the total potential of the disc and halo components. The disc rotation curve is given by the equation:

$v_{\mathrm{rot}}=\sqrt{R \frac{\mathrm{d} \Phi_{0}}{\mathrm{~d} R}}=\frac{v_{0} R}{\sqrt{R_{\mathrm{C}}^{2}+R^{2}}}$.
Equation (3) gives the rotational velocity of a collisionless disc if the velocity dispersion of the disc is equal to zero. In a disc with a non-zero velocity dispersion, the mean rotational velocity $\left\langle v_{\varphi}\right\rangle$ differs from the simple law given by Eq. (3) due to a collisionless "pressure" that influences the rotation of the disc.

The density distribution given by Eq. (1) is characterized by three parameters, namely the central surface density $\Sigma_{0} \exp (-\lambda)$, the radial scale length of the disc density distribution $R_{\mathrm{D}}$ (through $\lambda$ ), and the core radius $R_{\mathrm{C}}$. We use the core radius $R_{\mathrm{C}}$ and the asymptotic circular velocity $v_{0}$ as units to normalize the problem. The time unit is then determined by the ratio $R_{\mathrm{C}} / v_{0}$. The dimensionless parameter $S_{0}=G \Sigma_{0} R_{\mathrm{D}} / v_{0}^{2}$ gives the ratio of the mass of the disc to the total mass of the model within the radius $R \approx 5 R_{\mathrm{C}}$.

Following JH, we assume the distribution function of the disc particles in the form:

$$
\begin{aligned}
f(E, L)= & \Sigma_{0} \sum_{n=0}^{N}\left(\begin{array}{c}
N \\
n
\end{array}\right)\left(\frac{L}{R_{\mathrm{C}}}\right)^{2 n} g_{n}(E), \\
g_{n}(E)= & \frac{(-1)^{n+1}}{2^{n} \sqrt{\pi} \Gamma(n+1 / 2)} \\
& \times \frac{\mathrm{d}^{n+1}}{\mathrm{~d} E^{n+1}}\left(\mathrm{e}^{-2 N E / v_{0}^{2}} \mathrm{e}^{-\lambda \mathrm{e}^{E / v_{0}^{2}}}\right) .
\end{aligned}
$$

Here $E$ and $L$ are the total energy and the angular momentum of an individual star as

$E=\frac{1}{2}\left(v_{R}^{2}+v_{\varphi}^{2}\right)+\Phi_{0}(R), \quad L=R v_{\varphi}$

The family of distribution functions given by Eqs. (4) and (5) depends on an integer parameter $N$, that controls the fraction of near-circular orbits, and thus controls the disc velocity dispersion. The radial and azimuthal velocity dispersions determined as

$C_{R}=\sqrt{\left\langle v_{R}^{2}\right\rangle}, \quad C_{\varphi}=\sqrt{\left\langle v_{\varphi}^{2}\right\rangle-\left\langle v_{\varphi}\right\rangle^{2}}$

are small for relatively high values of $N$. We select a moderately cold disc characterized by the parameters $N=6, \lambda=R_{\mathrm{C}} / R_{\mathrm{D}}=$ 0.625 and $S_{0}=0.34$. The upper panel of Figure 1 shows the rotation curve $v_{\text {rot }}$ determined by Eq. (3), the mean rotational velocity $\left\langle v_{\varphi}\right\rangle$ and the normalized surface density $\mathrm{e}^{\lambda} \Sigma_{\mathrm{D}}(R) / \Sigma_{0}$ for this model. The lower panel of Fig. 1 shows Toomre's $Q$-parameter together with the radial and azimuthal velocity dispersions as a function of the radial distance $R$. For the selected model, the $Q$ parameter is greater than unity everywhere in the disc, and the model is stable for $m=0$ perturbations.

In the linear regime, the spiral density perturbation can be written in polar coordinates $(R, \varphi)$ as:

$\Sigma_{m}(R, \varphi, t)=\hat{A}_{m, 0}(R) \mathrm{e}^{\mathrm{i}(m \varphi-\omega t)}$,

where

$\omega=m \Omega_{\mathrm{p}}+\mathrm{i} s, \quad \hat{A}_{m, 0}(R)=A_{m}(R) \mathrm{e}^{\mathrm{i} \theta_{m}(R)}$.

Here $A_{m}(R), \theta_{m}(R), \Omega_{\mathrm{p}}$ and $s$ are the amplitude, phase, pattern speed and growth rate of the $m$ th global mode, respectively.

Figure 2 displays the results of the linear global modal analysis of our model. We find that the collisionless disc is unstable towards two, three and four-armed spirals. We have shown the contour plots of the unstable eigenmodes, together with the positions of the corotation (CR) and outer Lindblad (OLR) resonances. Pattern speeds $\Omega_{\mathrm{p}}$ and growth rates $s$ of all unstable modes are collected in Table 2 . 

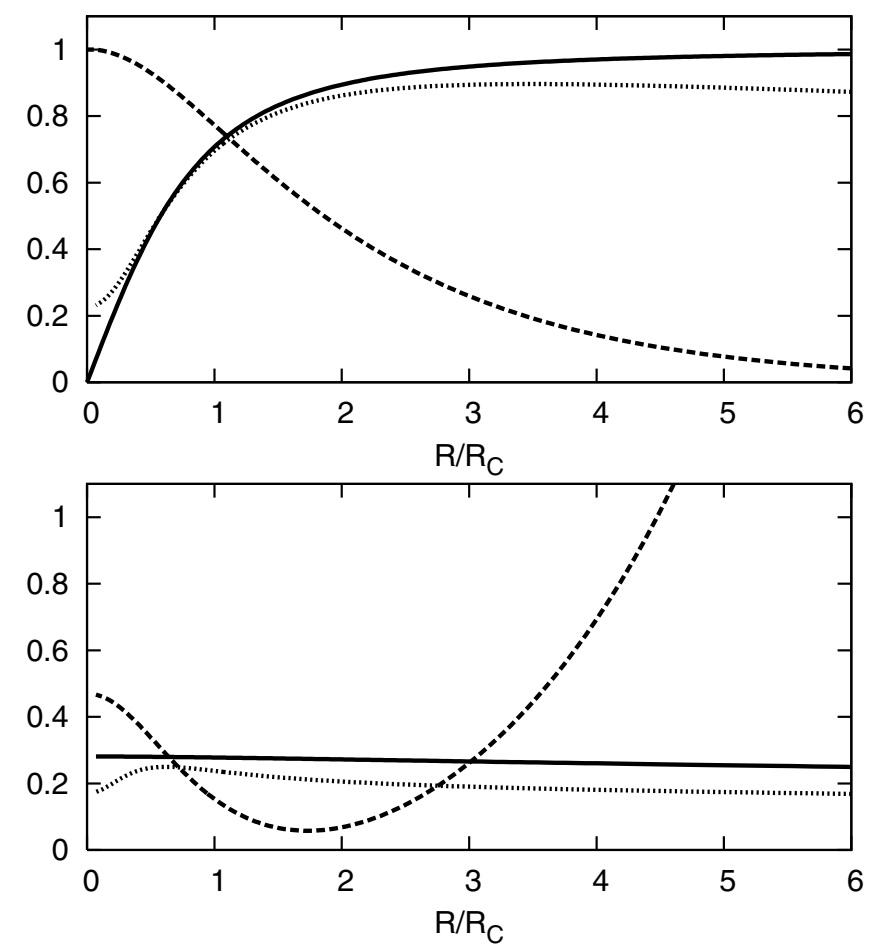

Fig. 1. Upper panel: solid line shows the rotation curve $v_{\text {rot }}$ of the model. Dotted line is the streaming velocity $\left\langle v_{\varphi}\right\rangle$ and dashed line is the normalized surface density distribution. Lower panel: solid and dotted lines show $C_{R}$ and $C_{\varphi}$, respectively. Dashed line is Toomre's $Q$ minus 1 $(Q-1)$. We have set the model parameters to $N=6, G \Sigma_{0} R_{\mathrm{D}} / v_{0}^{2}=0.34$ and $\lambda=R_{\mathrm{C}} / R_{\mathrm{D}}=0.625$.

Figure $2 \mathrm{a}$ shows the fastest growing $m=2$ bar-like mode ( $2 p$ in Table 2). The dimensionless pattern speed and growth rate for this mode are $\Omega_{\mathrm{p}}=0.768$ and $s=0.642$, respectively. Linear analysis also reveals another unstable $m=2$ global mode (Fig. 2b) that has a pattern speed of $\Omega_{\mathrm{p}}=0.443$ and a growth rate of $s=0.119$ ( $2 \mathrm{~s}$ in Table 2 ). This secondary mode is more spatially extended than the fundamental bar-mode, and occupies the disc within a few core radii. The spatial distribution of amplitude of the bar-mode has a single maximum located approximately at a distance of $R=0.25$ from the centre of the disc. The amplitude function of the secondary $m=2$ mode has three maxima of comparable amplitudes shifted approximately by 90 degrees with respect to each other. The secondary $m=2$ mode has also been shown in Fig. 9 of $\mathrm{JH}$, and we reproduce it here for completeness.

Figures $2 \mathrm{c}$ and $\mathrm{d}$ show the contour plots for the unstable $m=3$ and $m=4$ modes found in the linear stability analysis. These modes occupy approximately the same region of the disc as the secondary $m=2$ mode does. These modes, however, grow faster than the secondary $m=2$ mode (see Table 2 ). Therefore, the dynamics of perturbations in the outer regions of the disc is governed mostly by the $m=3$ and $m=4$ perturbations. The patterns of $m=3$ and $m=4$ instabilities freely extend up to the outer Lindblad radius. This shows that the corotation resonance has no influence on these modes.

\section{Numerical simulations}

\subsection{Code and data analysis}

Our $N$-body simulations are carried out with the help of the SUPERBOX code (Fellhauer et al. 2000). This is a highly efficient particle-mesh-code with nested and comoving grids, based on a leap-frog scheme with second order force calculation. Nested grids, comoving with the center of mass, allow us to achieve high resolution in the central parts of the collisionless systems. The code has been successfully applied to study mergers of galaxies (Madejsky \& Bien 1993), galaxy-satellite disruption (Klessen \& Kroupa 1998) and the orbital decay of satellite galaxies (Peñarrubia et al. 2002; Just \& Peñarrubia 2005). It was also used to study orbital evolution of a supermassive black hole in a galactic nucleus (Spinnato et al. 2003).

We use SUPERBOX to simulate the dynamics of perturbations in a collisionless disc, which is in rotational equilibrium under the influence of its self-gravity and the gravitational potential of an external rigid halo. The code is three dimensional. To study the dynamics of a two-dimensional gravitating disc, we confine the initial distribution of particles to the $(x, y)$-plane and set vertical velocities of the particles equal to zero. The dynamics of the model was simulated using different sets of the numerical parameters (i.e., number of particles, grid resolution, etc.) listed in Table 1. The radial extent of the inner, intermediate and the outer grid zones are determined by the parameters $R_{\mathrm{i}}, R_{\mathrm{m}}$ and $R_{\mathrm{o}}$, and the spatial resolution in each grid zone is determined for the inner grid zone by the parameter $d_{\mathrm{i}}$.

The SUPERBOX has a fixed time step, so to resolve the motion of the particles in the central regions of the disc, we use a time step of $\mathrm{d} t=0.05 \mathrm{Myr}$. This is less than a typical crossing time of a grid cell in the inner regions of the disc $(\approx 0.1 \mathrm{Myr})$.

The disc is built with equal mass particles. To construct a 2D collisionless model in dynamical equilibrium, we use the distribution function given by Eq. (4), and set velocities of the particles in $z$-direction and their $z$-coordinates equal to zero $\left(z_{j}=v_{z, j}=0\right)$. The gravitational potential of a rigid halo is assumed to be spherical and is calculated by subtracting the disc potential $\Phi_{\mathrm{D}}$ from the total potential given by Eq. (2).

To determine the macroscopic characteristics of the disc, as a function of radius, such as surface density $\Sigma(R)$, disc rotation $\left\langle v_{\varphi}\right\rangle$ and the radial and azimuthal velocity dispersions $C_{R}$ and $C_{\varphi}$, we divide the disc into $n_{r}=100$ rings of equal width and calculate the macroscopic values in each ring. Figure 3 shows the $N$-body realization of the initial equilibrium profiles of the disc. In this figure, the solid lines show the rotation velocity and the velocity dispersions of the disc, calculated with Eq. (4), and the points are the $N$-body realization of disc equilibrium. As it can be judged from Fig. 3, there is satisfactory agreement between the theoretical model and its $N$-body realization except for the very central regions of the disc.

To quantify the growth rates and the pattern speeds of the unstable modes, we calculate the Fourier components of the perturbed density for azimuthal wavenumbers $m=1,2, \ldots, 6$. With point-like particles, the Fourier components are given by the real part of the expression

$\tilde{\Sigma}_{m}=\tilde{A}_{m}\left(R_{i}\right) \mathrm{e}^{\mathrm{i} m \varphi}=\frac{M_{i}}{S_{i}} \sum_{j=1}^{N_{i}} \mathrm{e}^{\mathrm{i} m\left(\varphi-\varphi_{j}\right)}$

where $S_{i}$ is the area of the $i$ th ring element corresponding to $R_{i}, N_{i}$ is the number of particles in the $i$ th ring, and $M_{i}$ is the total mass of particles that lie on $S_{i}$. By defining the amplitude $A_{m}\left(R_{i}\right)=\left|\tilde{A}_{m}\left(R_{i}\right)\right|$ we get

$\Sigma_{m}=A_{m}\left(R_{i}\right) \cos \left(m \varphi-\theta_{m}\right)$.

Generally, the amplitude $A_{m}(R, t)$ and the phase $\theta_{m}(R, t)$ of the modes depend on time and radius. By measuring these 

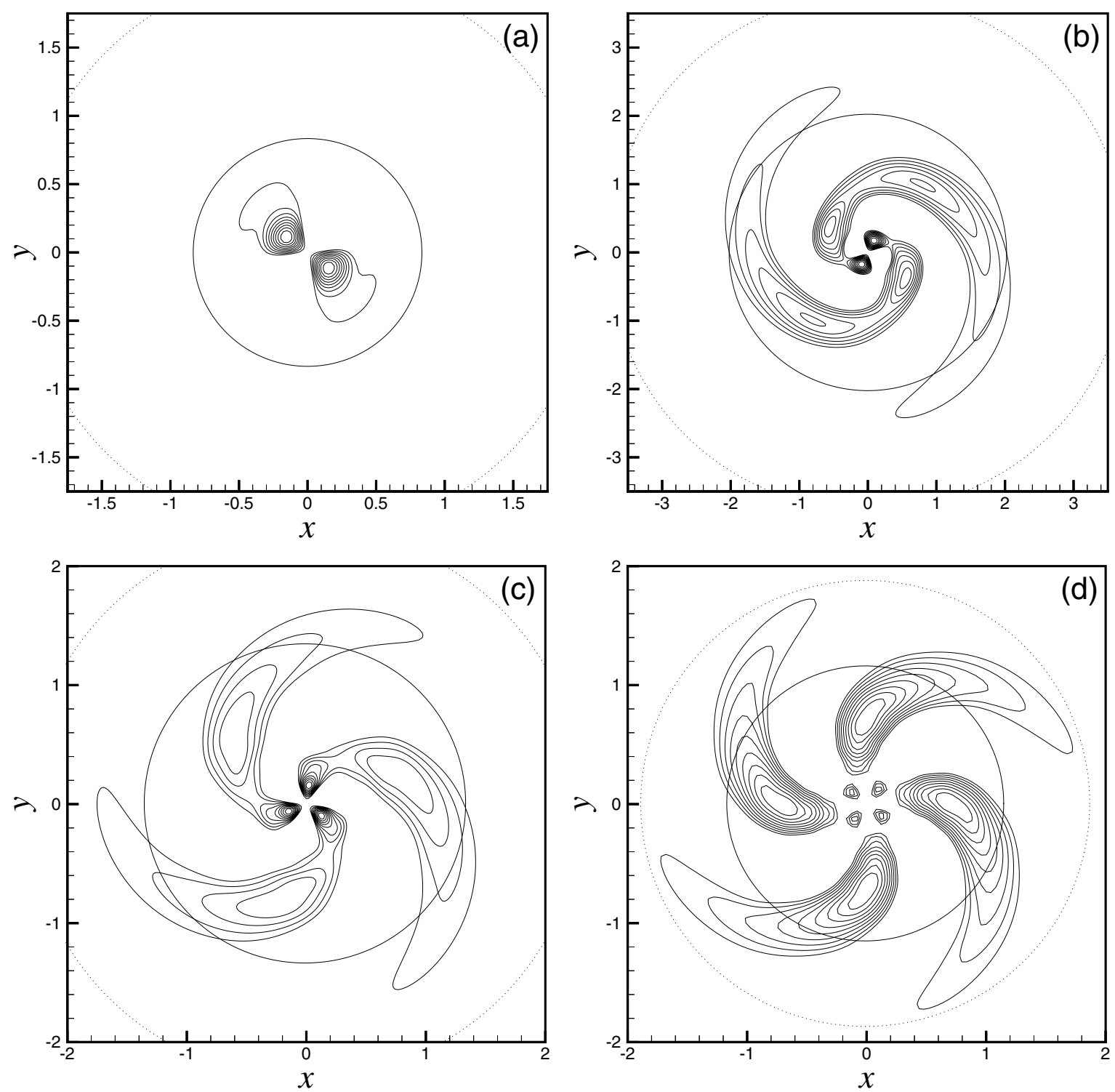

Fig. 2. Unstable modes of the cored exponential disc for $N=6, \lambda=R_{\mathrm{C}} / R_{\mathrm{D}}=0.625$ and $G \Sigma_{0} R_{\mathrm{D}} / v_{0}^{2}=0.34$. Contours show the positive surface density perturbations. The contour levels are equally spaced from 10 to 90 percent of the maximum. Solid and dotted circles mark the CR and OLR circles respectively. a) The fundamental $m=2$ mode. b) The secondary $m=2$ (spiral) mode. c) $m=3$. d) $m=4$.

quantities, we can estimate the growth rate $s$ and the pattern speed $\Omega_{p}$ of the modes by fitting the perturbed quantities to the expressions

$A_{m}(t) \rightarrow A_{m, 0} \mathrm{e}^{s t}, \quad \theta_{m}(t) \rightarrow \theta_{m, 0}+m \Omega_{\mathrm{p}} t$.

If the growth rate and the pattern speed of a Fourier component are independent of radius, the perturbation is dominated by an eigenmode.

To test the influence of the resolution effects and to determine the long-term behaviour caused by numerical errors, we simulated the dynamics of a stable model with a low disc mass (model A in Table 1). For this model, the number of particles as well as the grid resolution has been varied. We find that after adapting the particle distribution to a grid-based potential the disc stays in equilibrium. After $3 \mathrm{Gyr}$, the artificial heating caused by the particle-mesh scattering is lower than $5 \%$ of the initial velocity dispersion which shows that SUPERBOX is intrinsically collisionless.

To test the dynamics of a three-dimensional stellar disc, we expanded model A2 in the vertical direction to an isothermal slab with the scale height of $h=100 \mathrm{pc}$ (model A3). The result of this exercise is that the dynamical equilibrium is not affected by the thickening and that the stability of the disc is not destroyed.

\subsection{The unstable model}

In this section we discuss properties of the unstable modes found in numerical simulations, and compare the results to those obtained in a linear analysis. We find that the low-resolution model B0 leads to a significant discrepancy between $N$-body simulations and analytical predictions. We therefore discuss results obtained for the higher-resolution model B1 which is built with 13 million particles. Models B2-B8 differ from the model B1 in the numerical parameters, namely the outer cutoff radius, the time step, the number of particles, and the grid resolution, and serve to illustrate that parameter variations do not affect the results of $N$-body simulations for our fiducial model. Model B9 follows the disc evolution three times longer than B1 with the same parameters to investigate the nonlinear evolution of the unstable modes. Models B10-B12 are a series, where only 
Table 1. Parameters of the different models. All models have the same circular velocity $v_{0}=220 \mathrm{~km} \mathrm{~s}^{-1}$ and disc scale length $R_{\mathrm{D}}=3 \mathrm{kpc}$. Model A with $\lambda=R_{\mathrm{C}} / R_{\mathrm{D}}=1$ and $S_{0}=0.133$ is the stable reference model. Model B with $\lambda=0.625$ and a larger disc mass $S_{0}=0.329$ corresponds to the unstable model in Sect. 2. dim and $R_{\max }$ are the dimension and cutoff radius of the disc. $\Sigma_{0}$ determines the surface density of the disc (see Eq. (1)) and $\mathrm{d} t$ is the time step of the models. $N$ gives the number of particles, the next column the number of grid cells in each grid. $R_{\mathrm{i}}, R_{\mathrm{m}}$ and $R_{\mathrm{o}}$ are the radii of the inner, middle and outer grid. $d_{i}$ gives the cell size of the inner grid cells.

\begin{tabular}{|c|c|c|c|c|c|c|c|c|c|c|c|}
\hline "Model & $\overline{\text { dim }}$ & $\lambda$ & $\begin{array}{r}R_{\max } \\
\mathrm{kpc}\end{array}$ & $\begin{array}{r}\Sigma_{0} \\
M_{\odot} / \mathrm{pc}^{2}\end{array}$ & $\begin{array}{l}\mathrm{d} t \\
\mathrm{Myr}\end{array}$ & $\begin{array}{r}N \\
10^{6}\end{array}$ & $\overline{\text { grids }}$ & $\begin{array}{r}R_{\mathrm{o}} \\
\mathrm{kpc}\end{array}$ & $\begin{array}{r}R_{\mathrm{m}} \\
\mathrm{kpc}\end{array}$ & $\begin{array}{r}R_{\mathrm{i}} \\
\mathrm{kpc}\end{array}$ & $\begin{array}{l}\overline{d_{i}} \\
\mathrm{pc}\end{array}$ \\
\hline A0 & $2 \mathrm{D}$ & 1.0 & 16.0 & 500 & 0.05 & 4 & $64^{3}$ & 22.0 & 5.0 & 1.0 & 33.3 \\
\hline A1 & $2 \mathrm{D}$ & 1.0 & 16.0 & 500 & 0.05 & 4 & $128^{3}$ & 22.0 & 5.0 & 1.0 & 16.1 \\
\hline A2 & $2 \mathrm{D}$ & 1.0 & 16.0 & 500 & 0.05 & 13 & $128^{3}$ & 22.0 & 5.0 & 1.0 & 16.1 \\
\hline A3 & $3 \mathrm{D}$ & 1.0 & 16.0 & 500 & 0.05 & 13 & $128^{3}$ & 22.0 & 5.0 & 1.0 & 16.1 \\
\hline B0 & $2 \mathrm{D}$ & 0.625 & 16.0 & 1233 & 0.05 & 4 & $64^{3}$ & 17.0 & 4.0 & 1.0 & 33.3 \\
\hline B1 & $2 \mathrm{D}$ & 0.625 & 16.0 & 1233 & 0.05 & 13 & $128^{3}$ & 17.0 & 4.0 & 1.0 & 16.1 \\
\hline B2 & $2 \mathrm{D}$ & 0.625 & 16.0 & 1233 & 0.05 & 13 & $128^{3}$ & 17.0 & 4.0 & 1.0 & 16.1 \\
\hline B3 & $2 \mathrm{D}$ & 0.625 & 16.0 & 1233 & 0.05 & 13 & $128^{3}$ & 17.0 & 4.0 & 1.0 & 16.1 \\
\hline B4 & $2 \mathrm{D}$ & 0.625 & 16.0 & 1233 & 0.05 & 13 & $128^{3}$ & 17.0 & 4.0 & 1.0 & 16.1 \\
\hline B5 & $2 \mathrm{D}$ & 0.625 & 14.0 & 1233 & 0.025 & 13 & $128^{3}$ & 17.0 & 4.0 & 1.0 & 16.1 \\
\hline B6 & $2 \mathrm{D}$ & 0.625 & 12.0 & 1233 & 0.05 & 20 & $128^{3}$ & 17.0 & 4.0 & 1.0 & 16.1 \\
\hline B7 & $2 \mathrm{D}$ & 0.625 & 13.0 & 1233 & 0.05 & 40 & $128^{3}$ & 17.0 & 4.0 & 1.0 & 16.1 \\
\hline B8 & $2 \mathrm{D}$ & 0.625 & 13.0 & 1233 & 0.05 & 13 & $256^{3}$ & 17.0 & 4.0 & 1.0 & 7.9 \\
\hline B9 & $3 \mathrm{D}$ & 0.625 & 16.0 & 1233 & 0.05 & 13 & $128^{3}$ & 17.0 & 4.0 & 1.0 & 16.1 \\
\hline B10 & $2 \mathrm{D}$ & 0.625 & 12.0 & 1233 & 0.02 & 3 & $128^{3}$ & 17.0 & 4.0 & 1.0 & 16.1 \\
\hline B11 & $2 \mathrm{D}$ & 0.625 & 12.0 & 1233 & 0.02 & 13 & $128^{3}$ & 17.0 & 4.0 & 1.0 & 16.1 \\
\hline B12 & $2 \mathrm{D}$ & 0.625 & 12.0 & 1233 & 0.02 & 30 & $128^{3}$ & 17.0 & 4.0 & 1.0 & 16.1 \\
\hline
\end{tabular}

Table 2. Growth rates and pattern speeds of the different modes for model B12. The time unit is $R_{\mathrm{C}} / v_{0}=8.52 \mathrm{Myr}$.

\begin{tabular}{r|rrrr|rr}
\hline \hline Mode & \multicolumn{4}{|c}{ Analytical } & \multicolumn{3}{c}{ Simulations } \\
$m$ & $\Omega_{\mathrm{p}}$ & $s$ & $R_{\mathrm{CR}}$ & $R_{\mathrm{OLR}}$ & $\left\langle\Omega_{\mathrm{p}}\right\rangle$ & $\langle s\rangle$ \\
\hline $2 \mathrm{p}$ & 0.768 & 0.642 & 0.834 & 2.077 & 0.78 & 0.63 \\
$2 \mathrm{~s}$ & 0.443 & 0.119 & 2.042 & 3.775 & 0.61 & 0.41 \\
3 & 0.597 & 0.153 & 1.345 & 2.320 & 0.62 & 0.33 \\
4 & 0.653 & 0.164 & 1.159 & 1.880 & 0.57 & 0.24 \\
\hline
\end{tabular}

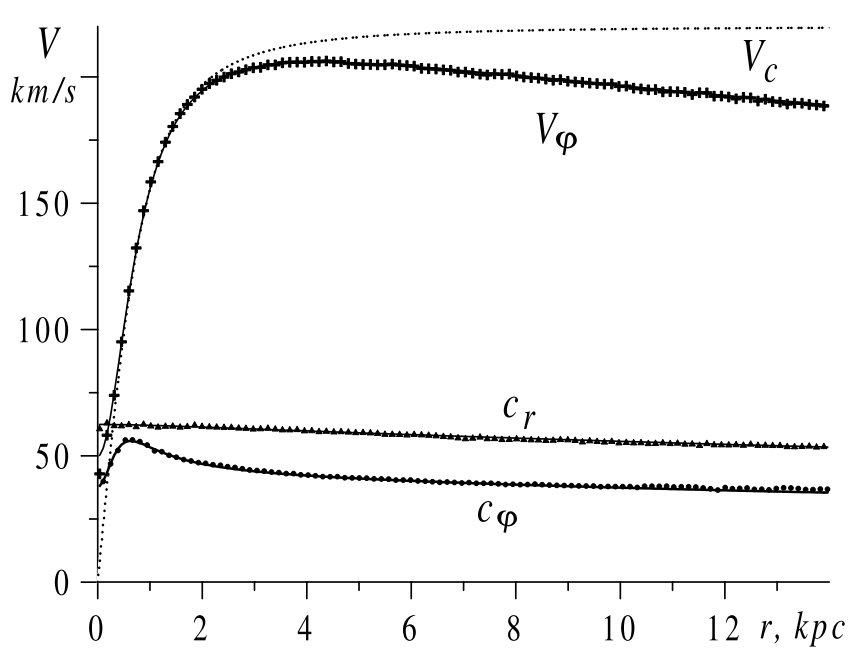

Fig. 3. The rotational velocity and velocity dispersions of the disc as a function of the radial distance $R$. Solid lines are the disk equilibrium profiles calculated with help of the distribution function (Eq. (4)). The points correspond to the $N$-body realizations of the initial equilibrium. Dotted line shows the rotation curve $v_{0}(R)$ of Eq. (3).

the number of particles is changed for the determination of the $N$ dependence of pattern speeds and growth rates.

Figure 4 shows a time sequence for the contour plots of the perturbed disc surface density plotted up to the nonlinear saturation phase. Linear analysis of our model predicts the existence of a few simultaneously growing unstable modes. The results of $N$-body simulations are in qualitative agreement with the linear analysis predictions. As expected, the early stages of disc evolution are governed by the fastest growing $m=2$ global barmode, developing in disc central regions. This mode is seen in Fig. 4 starting from $25 \mathrm{Myr}$. By $60 \mathrm{Myr}$, the central bar-mode saturates, and the perturbations continue to grow in the outer disc regions. The fastest growing mode is confined to the central kiloparsec of the disc, and the dynamics of the outer disc regions is governed by more slowly growing spirals. The disc dynamics in outer regions is determined by a superposition of slowly growing two-armed, three-armed and four-armed spirals. These modes are shown in Fig. 2. $\mathrm{N}$-body simulations confirm this finding.

For quantitative comparison of growing global modes found in $N$-body simulations with the analytically predicted results, we calculate a Fourier decomposition of the density perturbations. This allows us to determine the amplitude $A_{m}(r, t)$ and phase $\Theta_{m}(r, t)$ of an $m$-armed spiral mode as a function of time and of the disc radius, and to determine the growth rate and the pattern speed of each Fourier component as a function of radius. Figure 5 shows the amplitude and the phase of Fourier modes $m=2,3,4$ calculated at different radii in the disc. In the central regions, the dynamics of perturbations is governed by the fastest growing $m=2$ mode, which saturates at about $40 \mathrm{Myr}$. In the outer disc regions, the dynamics is dictated by the $m=3$ perturbation prevailing over its $m=2$, and $m=4$ competitors. As can be judged from Fig. 5, the spiral growth rates in the linear stage and their pattern speeds are fairly independent of radius, which indicates that perturbations are indeed the growing global modes. The growth rates $s$ and pattern speeds $\Omega_{\mathrm{p}}$ for the primary mode $m=2$ do not change value at the transition from the linear stage to the essential nonlinear stage $(t \gg 1 / s)$.

This is additionally illustrated in Fig. 6 showing the radial dependence of the growth rates, pattern speeds (upper frames), and the amplitudes of $m=2,3,4$ Fourier components as a function of radius. The parameters have been calculated from $N$-body simulations at time $40 \mathrm{Myr}$. The fastest growing 

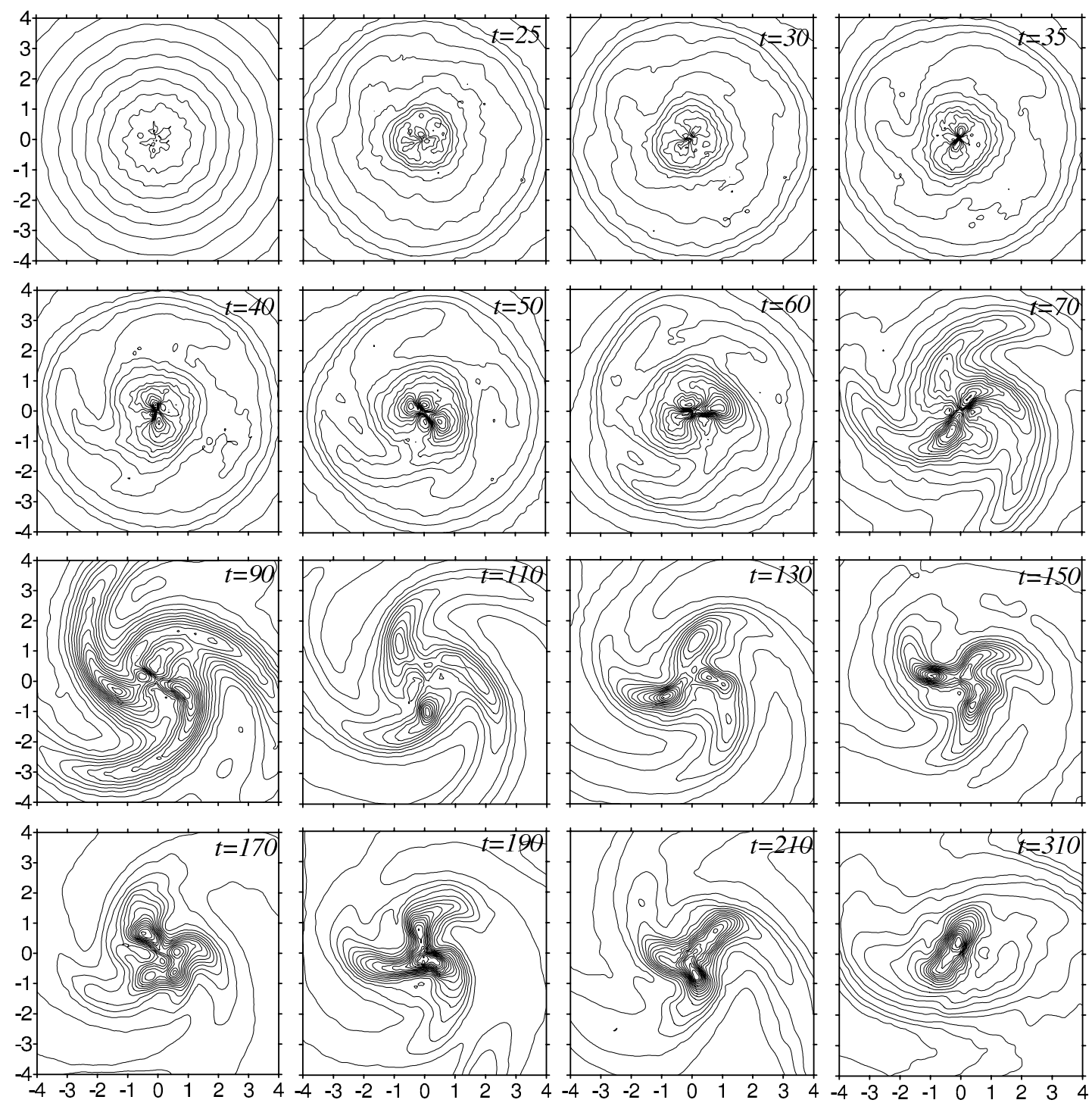

Fig. 4. Temporal evolution of the surface density distribution for an unstable disc (model shown in Fig. 2). A rapidly growing rotating bar-mode emerges in the central regions of the disc at early stages of disc evolution. At later times, the more slowly growing three-armed spiral determines the dynamics of perturbations in the outer regions of the disc. The time $t$ is given in Myr.

bar-perturbation grows as a whole in the central regions of the disc, and rotates with fairly constant angular velocity. The measured values for the growth rate and for the pattern speed for this mode agree within errors of measurement with the linear analysis. The pattern speed of the bar-mode of $\Omega_{p}=0.78$ agrees with the theoretical value of $\Omega_{\mathrm{p}}=0.77$, and the growth rates found in both linear analysis and $N$-body simulations quantitatively agree as well. The amplitude of the bar-mode reaches its maximum at about $0.25 \mathrm{kpc}$ (bottom frame of Fig. 6), and then decreases with radius. Such a radial profile agrees qualitatively with the linear analysis predictions (solid line). A discrepancy between the linear analysis and numerical simulations in the outer regions of the disc is explained by the presence of a secondary $m=2$ global mode.

The agreement between growth rates and pattern speeds for $m=3$, and $m=4$ modes measured in $N$-body simulations and those determined in linear analyses are less satisfactory. While pattern speeds agree reasonably well, the growth rates measured in $N$-body simulations show a considerable mismatch with the values found in linear analyses. Being lower than the growth rate of the fastest growing bar-mode, the growth rates in $N$-body simulations are higher than the analytical predictions. In part, the discrepancy can be related to the still low resolution of our simulations which is not high enough to properly model multi-modal behaviour of the unstable disc.

Figure 7 shows the dependence of the growth rate and the pattern speed of the principal bar-mode as a function of the number of particles used in $N$-body simulations. As can be seen from Fig. 7, the parameters of the unstable mode depend strongly on resolution, i.e., number of particles used in $N$-body simulations. The experiment with 3 million particles gives a large discrepancy between the predicted parameters of the unstable mode (solid lines in Fig. 7) and the values measured in $N$-body simulations. A simulation with 13 million particles gives better agreement with theory, and one with 30 million particles gives a satisfactory agreement. Additionally, SUPERBOX achieves best resolution 

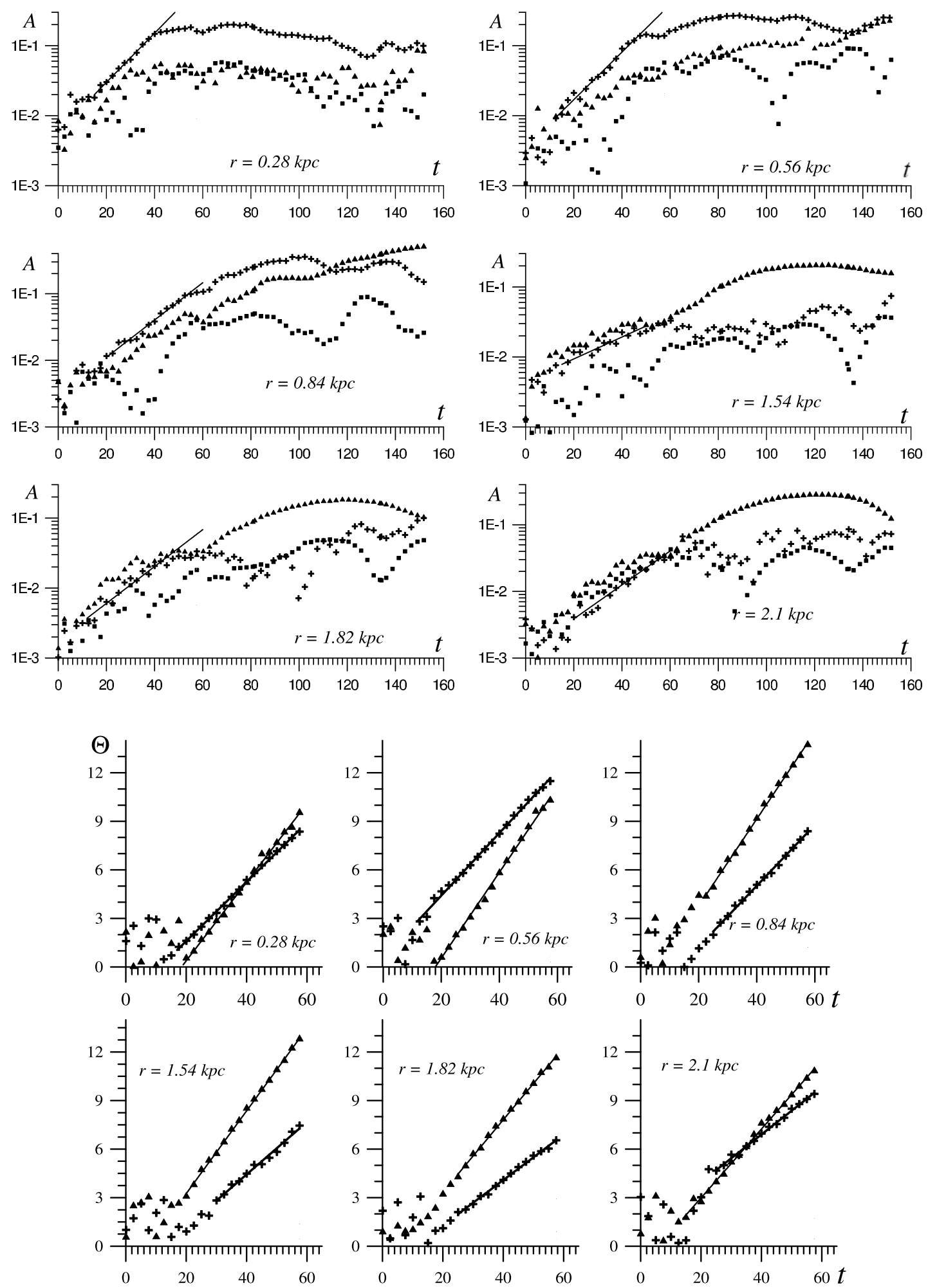

Fig. 5. The dependence on time of $m=2$ (crosses), $m=3$ (triangles), and $m=4$ (squares) amplitudes of the global modes $A$, measured at different radii. In the central regions of the disk the fastest growing is the $m=2$ global bar-mode. At the disc periphery the three armed perturbation prevails over other competitors. The time evolution for the phases of $m=2$ (crosses) and $m=3$ (triangles) Fourier components $\Theta$ is shown in the lower frames.

in the disc's central regions, so the parameters of the centrally confined fastest growing bar-mode are in better agreement with the analytical predictions compared to the more slowly growing modes developing in the outer regions of the disc. An increase of particle resolution in $\mathrm{N}$-body simulations should lead to a better agreement of the parameters of the secondary unstable modes with theory.
The spatial appearance of the eigenmodes is determined by the radial dependence of the amplitude and the phase of each Fourier component.

The $m=2$ Fourier component is a superposition of the primary and of the secondary unstable modes. We could not achieve a reliable decomposition of the two $m=2$ modes because of the noise and the nonlinear effects. Nevertheless, it is possible to 

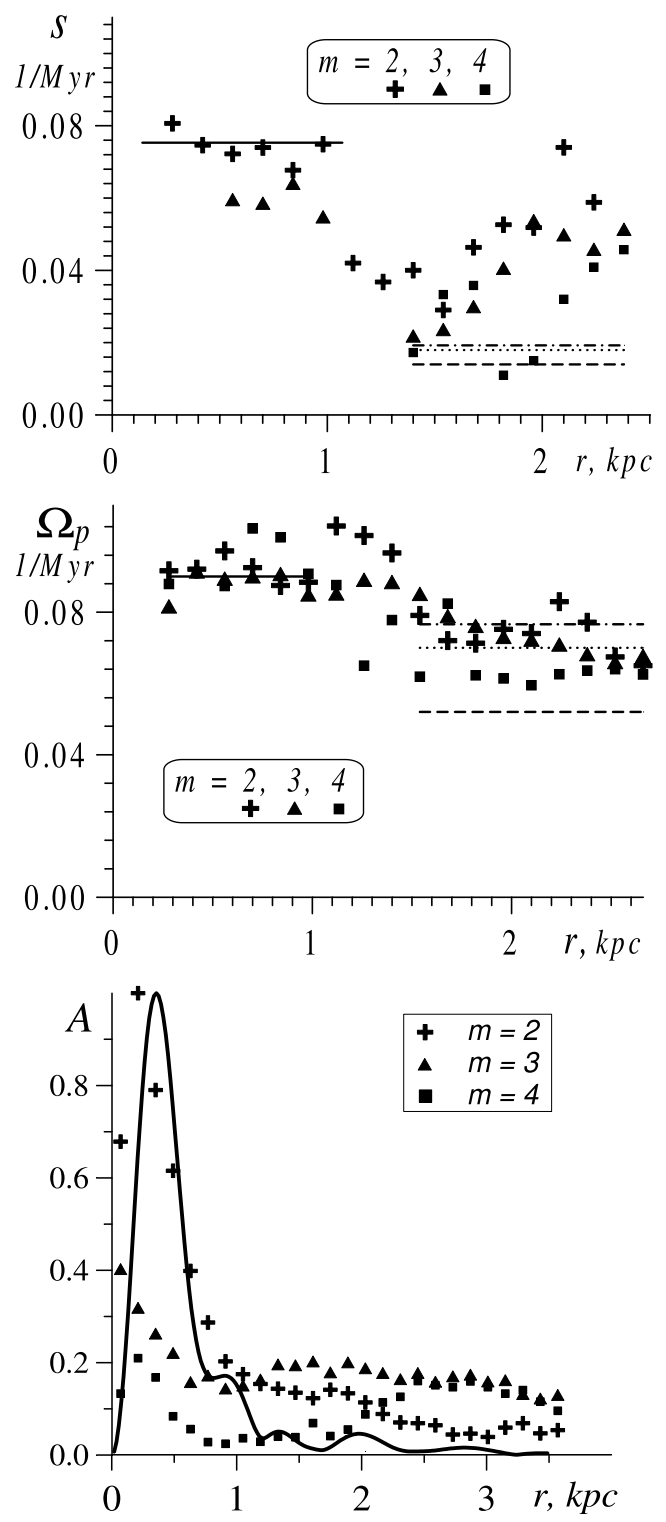

Fig. 6. Upper and middle panels: growth rates and pattern speeds for $m=2, m=3$ and $m=4$ Fourier components measured at different radii. In the central regions of the disc, the growth rate and pattern speed of the $m=2$ Fourier component agree with the linear analysis prediction of the bar mode (solid lines). Analytic prediction of the growth rates and pattern speeds are indicated at larger radii for $m=2$ (dashed lines), $m=3$ (dotted lines) and $m=4$ (dot-dashed lines) Fourier components. Lower panel: points - the amplitudes of $m=2,3$ and 4 - Fourier components as a function of radius taken from $N$-body simulations at time 40 Myr. The amplitude profile for the most unstable $m=2$ central bar-mode agrees qualitatively well with the linear analysis (solid line).

estimate which of the two $m=2$ modes is dominant in a particular region of the disc.

The unstable modes developing in $N$-body simulations agree qualitatively with the linear analysis not only in the growth rate and the pattern speed, but also in their spatial appearance. Figure 8 shows the contour plots of the surface density for the $m=2,3$ and 4 Fourier components as determined from $N$-body simulations (top frames) compared to the surface density contour plots for the unstable modes calculated by a linear stability analysis. For comparison, only the secondary $m=2$ linear mode is shown in the bottom left frame of Fig. 8a. As one can see, the
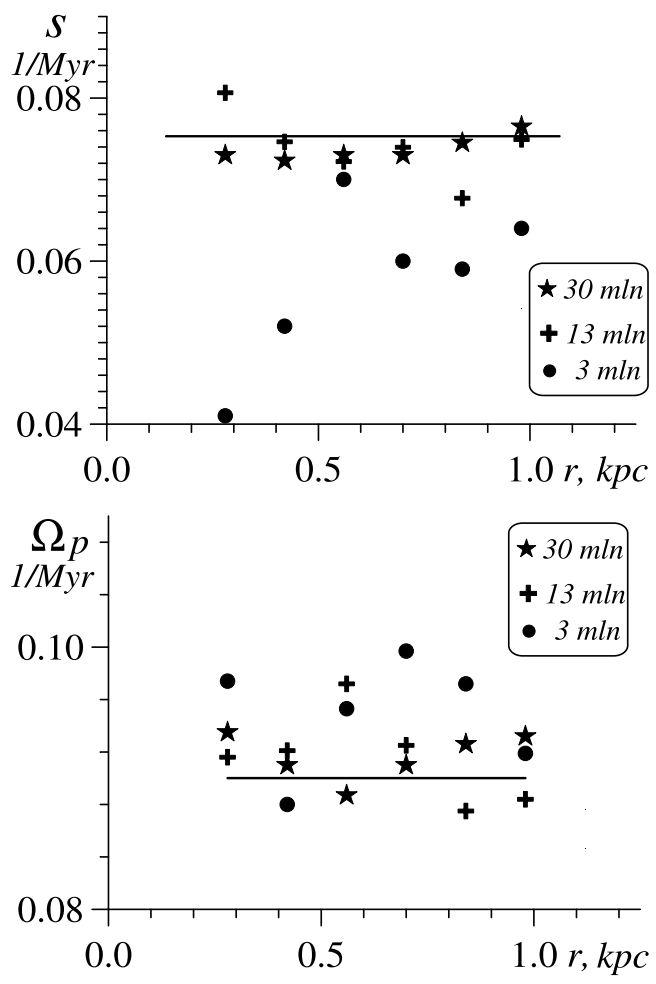

Fig. 7. The growth rate and the pattern speed of the principal $m=2$ bar-mode as a function of a number of particles in $N$-body simulations.

spatial range, and winding of the unstable modes agree qualitatively in both $N$-body and linear predictions.

Figure $8 \mathrm{~b}$ shows a comparison of spatial distributions of perturbations within the central half-kiloparsec of the disc. The density distribution for the central bar-mode agrees with the analytically predicted density distribution plotted in the lower left panel. An agreement for density distributions of $m=3$, and $m=4$ modes is also qualitatively acceptable but the details are different because of the limited resolution of the $\mathrm{N}$-body model discussed above.

\section{Summary}

We use high resolution $N$-body simulations to follow the dynamics of growing spiral perturbations that develop in a collisionless disc from the initial noise perturbations. At least ten million particles with a minimum grid resolution of $128^{3}$ are needed to reach a robust accuracy level so that the evolution of perturbations is no longer dominated by noise.

Comparison of $N$-body simulations with the results of linear stability analysis shows an agreement between both approaches. The most unstable global bar-mode developing from the noise perturbations has a pattern speed and a growth rate as predicted by theory. Other unstable modes (the primary $m=3, m=4$ modes, and a weak secondary $m=2$ mode) qualitatively agree with the theoretical results. However the pattern speeds and growth rates of the secondary two-armed spiral, the three-armed and four-armed spirals are in lesser agreement with the theoretical values due to a still low resolution of the disc dynamics in outer regions.

We have demonstrated that a fast particle-mesh code SUPERBOX is useful to follow a detailed dynamics of a collisionless stellar disc. Due to the large number of particles which can be used in SUPERBOX simulations, the noise level, and the 

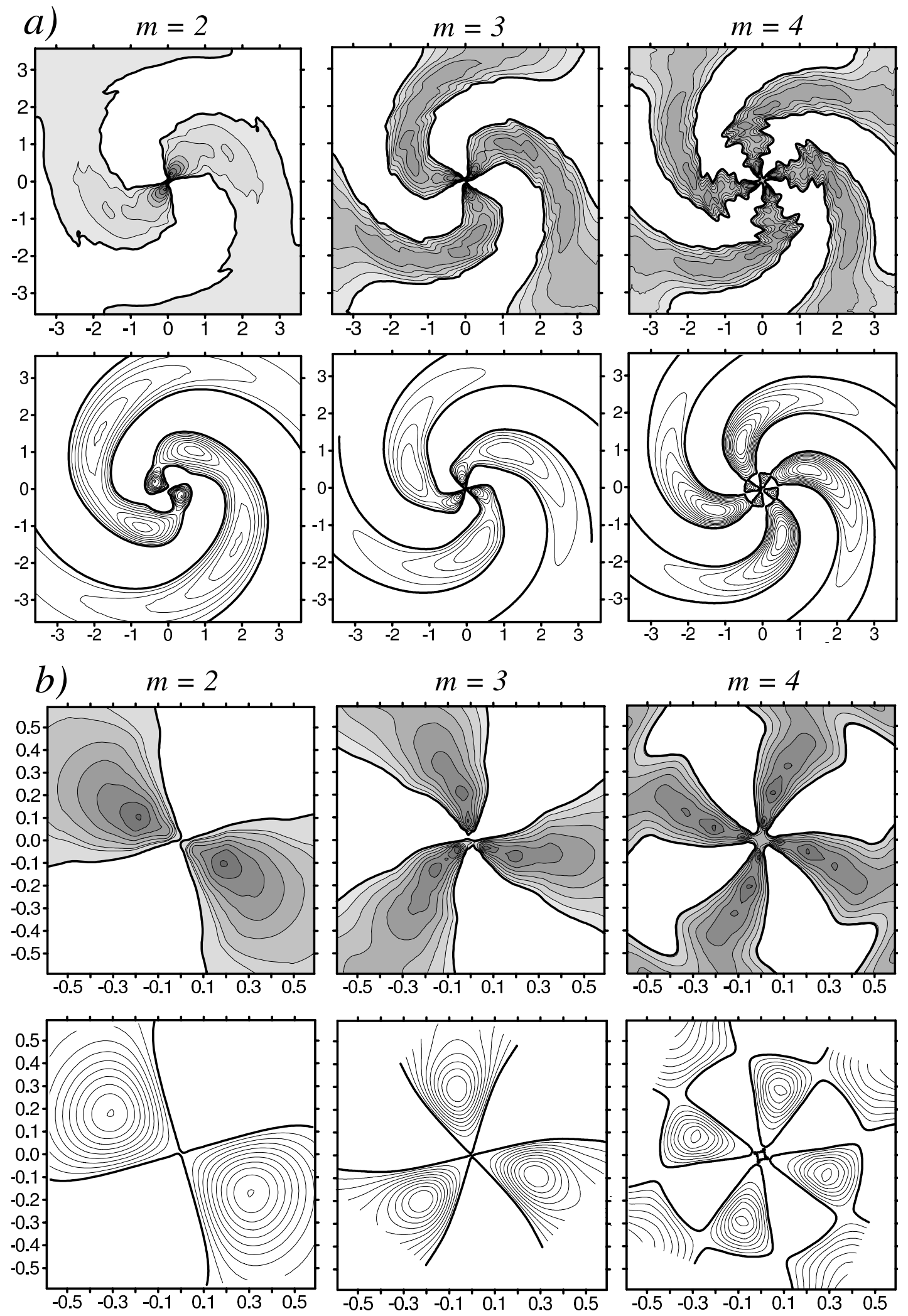

Fig. 8. a) Top frames - the surface density distribution for $m=2,3$ and 4 Fourier components determined from $N$-body simulations as compared to the surface density contour plots for the unstable modes determined from a linear stability analysis (bottom frames). For $m=2$ the secondary mode is shown (lower left). b) Density distributions within a central half-kiloparsec for the Fourier components shown in Fig. 8a. Here the primary $m=2$ mode is plotted at the lower left for comparison. 
numerical heating can be reduced to an insignificant level which allows us to follow the complex dynamics of a collisionless stellar disc.

Theoretical models of $\mathrm{JH}$ are two dimensional, and have a finite mean rotation at the disc centre due to a strong positive gradient of azimuthal velocity dispersion. Stability properties of the disc strongly depend on the inner boundary conditions of the disc. We plan to explore new theoretical models that have more realistic equilibrium properties.

Acknowledgements. We thank Rainer Spurzem, Peter Berczik and Toshio Tsuchyia for their contributions to the project, for their help in running the models and for lots of hints and clarifying discussions. We thank the Deutsche Forschungsgemeinschaft for supporting this project by DFG 436 RUS 17/10/03. A.V.K. also acknowledges the RFBR (07-02-01204).

\section{References}

Adams, F. C., Ruden, S. P., \& Shu, F. H. 1989, ApJ, 347, 959 Asghari, N. M., \& Jalali, M. A. 2006, MNRAS, 373, 337 Athanassoula, E., \& Sellwood, J. A. 1986, MNRAS, 221, 213 Bertin, G., Lin, C. C., Lowe, S. A., \& Thurstans, R. P. 1989a, ApJ, 338, 78 Bertin, G., Lin, C. C., Lowe, S. A., \& Thurstans, R. P. 1989b, ApJ, 338, 104 Evans, N. W., \& Read, J. C. A. 1998a, MNRAS, 300, 83
Evans, N. W., \& Read, J. C. A. 1998b, MNRAS, 300, 106 Fellhauer, M., Kroupa, P., Baumgardt, H., et al. 2000, NewA, 5, 305 Jalali, M. A., \& Hunter, C. 2005, ApJ, 630, 804 (JH)

Just, A., \& Peñarrubia, J. 2005, A\&A, 431, 861

Kalnajs, A. J. 1972, ApJ, 175, 63

Klessen, R. S., \& Kroupa, P. 1998, ApJ, 498, 143

Laughlin, G., Korchagin, V., \& Adams, F. C. 1997, ApJ, 477, 410

Laughlin, G., Korchagin, V., \& Adams, F. C. 1998, ApJ, 504, 945

Lin, C. C., \& Shu, F.H. 1964, ApJ, 140, 646

Lynden-Bell, D., \& Kalnajs, A. J. 1972, MNRAS, 157, 1

Madejsky, R., \& Bien, R. 1993, A\&A 280, 383

Miyama, S.M., et al. 1994, in Numerical Simulations in Astrophysics, ed. J. Franco, S. Lizano, L. Aguilar, \& E. Daltabuit (Cambridge: Cambridge Univ. Press), 305

Nelson, A. F., Benz, W., Adams, F. C., \& Arnett, D. 1998, ApJ, 502, 342

Peñarrubia, J., Kroupa, P., \& Boily, C.M. 2002, MNRAS, 333, 779

Peñarrubia, J., Just, A., \& Kroupa, P. 2004, MNRAS, 349, 747

Savonije, G. J., \& Heemskerk, M. H. M. 1990, A\&A, 240, 191

Sellwood, J. A. 1983, J. Comp. Phys., 50, 337

Sellwood, J. A., \& Athanassoula, E. 1986, MNRAS, 221, 195

Spinnato, P. F., Fellhauer, M., \& Portegies Zwart, S.F., 2003, MNRAS, 344, 22

Tomley, L., Steiman-Cameron, T. Y., \& Cassen, P. 1994, ApJ, 422, 850

Toomre, A. 1981, in Structure and Evolution of Normal Galaxies, ed. S.M. Fall \& D. Lynden-Bell (Cambridge, Cambridge Univ. Press), 111

Vauterin, P., \& Dejonghe, H. 1996, A\&A, 313, 465

Woodward, J. W., Tohline, J. E., \& Hachisu, I. 1994, ApJ, 420, 247

Zang, T.A. 1976, Ph.D. Thesis, Massachussetts Institute of Technology, Cambrigde, MA 